\title{
The Confine-and-Release Method: Obtaining Correct Binding Free Energies in the Presence of Protein Conformational Change
}

\author{
David L. Mobley $^{1}$, John D. Chodera ${ }^{2}$, and Ken A. Dill ${ }^{1^{*}}$ \\ 1 Department of Pharmaceutical Chemistry, University of California at San Francisco, San Francisco, CA \\ 94158-2517. \\ 2 Graduate Group in Biophysics, University of California at San Francisco, San Francisco, CA 94158-2517.
}

\begin{abstract}
Free energy calculations are increasingly being used to estimate absolute and relative binding free energies of ligands to proteins. However, computed free energies often appear to depend on the initial protein conformation, indicating incomplete sampling. This is especially true when proteins can change conformation on ligand binding, as free energies associated with these conformational changes are either ignored or assumed to be included by virtue of the sampling performed in the calculation. Here, we show that, in a model protein system (a designed binding site in T4 Lysozyme), conformational changes can make a difference of several $\mathrm{kcal} / \mathrm{mol}$ in computed binding free energies, and that they are neglected in computed binding free energies if the system remains kinetically trapped in a particular metastable state on simulation timescales. We introduce a general "confineand-release" framework for free energy calculations that accounts for these free energies of conformational change. We illustrate its use in this model system by demonstrating that an umbrella sampling protocol can obtain converged binding free energies that are independent of the starting protein structure and include these conformational change free energies.
\end{abstract}

\section{Keywords}

Confine-and-Release; binding free energy; free energy calculations; conformational change; alchemical free energy

Computational tools are becoming increasingly important in drug discovery ${ }^{1}$. A major goal is to use these methods to predict (absolute or relative) protein-ligand binding free energies. A great deal of effort $2,3,4$ has been focused on identifying which protein structures (i.e. apo, holo, or optimized in some manner) work best for estimating binding affinities. This emphasis on a single bound structure or conformation begs the question, "Can protein-ligand binding free energies be accurately predicted only a single protein conformation, or only some of the relevant protein conformations, are considered?" We demonstrate here that the answer is a decisive no in at least the model system considered here. There can be significant strain energies and free energy costs associated with trapping a protein into any metastable state, and, as we show here, the neglect of these costs can lead to substantial errors that depend on the metastable state chosen. (Here, we will use the term "structure" to refer to a single static structure, and the term "metastable state" to refer to a favorable region of configuration space (set of structures) that is kinetically distinct from other such regions).

\footnotetext{
*Author to whom correspondence should be addressed. E-mail: dill@ maxwell.compbio.ucsf.edu.
} 
Computed binding free energies are often sensitive to the starting protein structure, even with alchemical free energy methods $5,6,7,8,9,10$, which should not be the case if these simulations are converged. We believe this is for a similar reason: Even if full protein flexibility is allowed, the full range of relevant protein states may not be accessible on simulation timescales. This means that the protein is kinetically trapped in a particular metastable state, and the free energy cost of this trapping is neglected. Here, the problem is fundamentally a kinetic one: Large energy barriers can separate metastable protein states and trap the protein in a single metastable state on simulation timescales. Unfortunately, this trapping is inevitable whenever energy barriers are sufficiently large ${ }^{11}$, yet inadequate sampling even at the level of a single sidechain rotameric state can lead to a difference in several $\mathrm{kcal} / \mathrm{mol}$ in computed binding free energies 8 . The problem is that it is necessary to adequately sample multiple relevant protein metastable states, including at least the metastable states containing both the apo and holo structures.

Here, we describe a framework we call "confine-and-release" for computing absolute binding free energies that correctly accounts for multiple relevant metastable states, such as protein conformational changes on ligand binding. The framework is general, in that it may be implemented in a number of different ways. We demonstrate the framework in a model binding site using one particular approach based on umbrella sampling, below.

In this work, we will refer to the problem of kinetic trapping or confinement as "[virtual] confinement", to distinguish it from real confinement, where an external biasing potential is used. The confine-and-release approach discussed here can deal with both cases, but we illustrate it here with virtual confinement.

The basic theory underlying absolute binding free energy calculations has previously been described in detail (for example, in ${ }^{12,13}$ ). The absolute binding free energy is given as:

$$
\Delta G_{\text {bind }}=-k_{B} T \ln \frac{C^{o}}{8 \pi^{2}} \frac{\sigma_{P} \sigma_{L}}{\sigma_{P L}} \frac{Z_{P L}}{Z_{P} Z_{L}}+P^{o} \Delta V_{P L}
$$

where the protein-complex partition function is given by

$$
Z_{P L}=\int_{\text {complex }} e^{-\beta U(\vec{r})} d \vec{r}
$$

which is an integral over all of the protein-ligand conformations defining the bound state, and $Z_{P}$ and $Z_{L}$ are the corresponding partition functions consisting of integrals for the protein and ligand alone in solvent, respectively. $C^{o}$ denotes the standard concentration $(1 \mathrm{M})$, and the $\sigma$ factors are the symmetry numbers for the protein, for the ligand, and for the complex. These terms, as well as the $P^{o} \Delta V_{P L}$ pressure-volume work term, relate to the standard state, and are explained in detail elsewhere ${ }^{14}$.

The essential point here is that evaluating the binding free energy necessarily involves integrating over all of the relevant (low potential energy) conformations of the protein and ligand, including all metastable states. If that integration is incomplete, as in the case of inadequate sampling, the quantity calculated will not be a true binding free energy. In such cases of kinetic trapping, the free energy that is calculated can be called a "confined" binding free energy - it measures the binding free energy of the system [virtually] confined to a metastable state (for example, the region corresponding to the holo structure), and hence neglects certain components of the true binding free energy such as strain energies. This observation is related to that made earlier by Straatsma and McCammon in the context of salvation for molecules with multiple relevant rotameric states: Unless all relevant metastable states are sampled in some manner, computed free energies are "unreasonable" and incorrect 15 . 
We illustrate the problem with the example of $p$-xylene binding in a simple apolar cavity (an engineered cavity in T4 lysozyme) studied computationally by Deng and Roux ${ }^{8}$. Here, a single valine sidechain reorients upon ligand binding (as seen by comparing the apo and holo structures ${ }^{19}$ ).

We use simulation protocols employed previously ${ }^{14}$ with minor modifications described in the Supporting Information. These modifications involve improved parameters for the Particle mesh Ewald 16 treatment of long-range electrostatics, addition of a separate vacuum calculation in order to finish the cycle for computing binding free energies, and addition of a long-range correction term to account for attractive dispersion interactions between the ligand and protein that are neglected when simulations are run with a short cutoff. Very briefly, the overall procedure involves first restraining the ligand in complex, then annihilating the ligand's electrostatic interactions, followed decoupling its Lennard-Jones interactions. The restraints are then analytically removed and this is equivalent to having a protein with no ligand, plus a non-interacting, neutral ligand in solvent. The ligand electrostatic interactions are then restored in solvent, completing the thermodynamic cycle. The free energy of making each of these transformations is computed using free energy methods with a series of separate simulations at different alchemical intermediate states ( $\lambda$ values).

We start from the apo structure. We observe that the system remains trapped in the metastable state containing that structure over the course of all equilibration and production trajectories involved in the free energy calculation ( $1.11 \mathrm{~ns}$ at each $\lambda$ value). The resulting computed binding free energy is $-2.96+/-0.06 \mathrm{kcal} / \mathrm{mol}$ (where the uncertainty represents one standard deviation over a set of block bootstrap trials as described in the Supporting Information and previously ${ }^{14}$ ). If, instead, we start from the holo structure, we compute a binding free energy of $-7.27+/-0.09 \mathrm{kcal} / \mathrm{mol}$. If we examine the valine sidechain $\chi_{1}$ dihedral angle as a function of time for every simulation in these free energy calculations, we find that, in each case, it remains in its initial rotameric state. The valine does not cross its torsional energy barrier on simulation timescales. This causes significant errors: One computed binding free energy indicates $p$-xylene is a millimolar binder; the other indicates it is a micromolar binder.

We solve this problem using the "confine-and-release" framework, depicted in the thermodynamic cycle in Figure 1; there, confinement (in this case virtual confinement) is illustrated by a paperclip. We begin by recognizing that our calculated free energies are "confined" binding free energies, that is, free energies for binding of the ligand to a protein that is restricted to a particular metastable state. Then, to compute the true binding free energy, we must add the free energy of confining the protein to that metastable state when no ligand is bound and the free energy of releasing the protein from its confinement when the ligand is bound. Hence $\Delta G_{\text {bind }}^{o}=\Delta G_{\text {conf }}+\Delta G_{\text {bind, } C}^{o}+\Delta G_{\text {rel. }}$ In this expression, $\Delta G_{\text {bind }}^{o}$ is the true (standard) binding free energy; $\Delta G_{\text {bind, } C}^{o}$ is the standard binding free energy of the ligand to the confined protein; $\Delta G_{\text {conf }}$ is the free energy of confining the protein to this smaller region of configuration space in the unbound state; and $\Delta G_{r e l}$ is the free energy of releasing the protein from conformational confinement in the bound state ${ }^{1}$. This can be thought of as a generalization of conformational biasing potentials ${ }^{8}$.

Free energies of confinement and release can be computed using a variety of different algorithms. Here, since there is a single relevant degree of freedom that needs to be sampled, we employed umbrella sampling ${ }^{17}$. We computed the potential of mean force (PMF) for

\footnotetext{
${ }^{1}$ The two binding free energies here have superscripts indicating that they are "standard" binding free energies ${ }^{12}$ (i.e. at standard concentration), while the other two free energies do not, because these are conditional on the presence (release free energy) or absence (confining free energy) of the ligand, and hence do not depend on concentration. All simulations are conducted at standard pressure and at $300 \mathrm{~K}$.
} 
rotating the sidechain of Val111 throughout its range of motion in both the bound and unbound states (Fig. 2) (details available in the Supporting Information). From the PMF, we computed the free energy of confining the sidechain to each rotameric state (as described in the Supporting Information). To test reproducibility of the corrected true free energy, the entire confine-andrelease procedure was performed twice: Once using the apo structure and the associated metastable state (beginning from the apo crystal structure) for the binding calculation, and once using the holo structure (and metastable state) for the binding calculation. The same framework applies in either case.

Using the apo metastable state, we compute a confinement free energy in the unbound state of $0.01+/-0.04$ and a release free energy of $-0.6+/-0.1 \mathrm{kcal} / \mathrm{mol}$ in the bound state. Combining this with the computed confined binding free energy of $-2.96+/-0.06 \mathrm{kcal} / \mathrm{mol}$ yields a total binding free energy of $-3.5+/-0.2 \mathrm{kcal} / \mathrm{mol}$. Alternatively, using the holo metastable state, the confinement free energy is $4.2+/-0.2 \mathrm{kcal} / \mathrm{mol}$ and the release free energy is $0.28+/-0.08 \mathrm{kcal} /$ mol, which, when added to the computed confined binding free energy of $-7.27+/-0.09 \mathrm{kcal} /$ mol, yields a total binding free energy of $-3.3+/-0.2 \mathrm{kcal} / \mathrm{mol}$. The difference between the total binding free energies computed from the different crystal structures is now only $0.2+/$ $-0.3 \mathrm{kcal} / \mathrm{mol}-$ statistically indistinguishable from zero. Hence, we believe these values now represent the overall binding free energy, corrected for inadequate sampling of Val111. In this case, the experimental binding free energy is $-4.67+/-0.06 \mathrm{kcal} / \mathrm{mol}$, so our approach substantially improves agreement with experiment, especially when beginning from the holo structure.

Figure 2 shows that, for $p$-xylene, a single rotameric state dominates when the ligand is absent (Figure $2 \mathrm{a}$ ), and a different rotameric state dominates when the ligand is present (Figure 2b), although in (2b), both rotameric states are relevant - that is, both states contribute significant fractions to the free energy. In general, the relevant rotameric state may differ in the presence and absence of the ligand, or there may be multiple relevant states in either case.

Previous work on this binding site, beginning from the holo structure for each ligand, produced binding free energies that were 2.05 to $4.40 \mathrm{kcal} / \mathrm{mol}$ too negative relative to experiment ${ }^{8}$ for those compounds where Val111 reorients on ligand binding ( $p$-xylene, $o$-xylene, and $n$ butylbenzene, isobutylbenzene $)^{19}$. Indeed, these compounds were essentially the worst outliers in that study. Here, due to kinetic trapping, we had to apply a positive correction of $3.9 \mathrm{kcal} / \mathrm{mol}$ for $p$-xylene beginning from the holo structure. Though the previous work used a different force field and parameters, it seems likely that kinetic trapping of Val111 can explain a significant portion of the observed errors there. For example, if we applied our correction to their calculated value for $p$-xylene $(-9.06 \mathrm{kcal} / \mathrm{mol})$, the resulting binding free energy would be $-5.06 \mathrm{kcal} / \mathrm{mol}$ (calculated) versus $-4.67 \mathrm{kcal} / \mathrm{mol}$ (experiment).

Here, the confine-and-release technique was applied to a single degree of freedom. As other situations will undoubtedly require careful sampling of more than a single (known) degree of freedom, the calculation of free energies of confinement and release from potentials of mean force is not necessarily a general strategy for applying this framework. Rather, the key points here, are: First, correct binding free energies can only be obtained when protein conformational change is correctly accounted for. Second, protein conformational change contributes substantially to the overall binding free energy, even for changes as small as the reorientation of single sidechains. Thus, protein conformational changes should not simply be ignored in binding free energy calculations.

To compute confine-and-release free energies technique with the umbrella sampling approach discussed here, there are several requirements. First, one must know (i.e. crystallographically) or be able to predict (i.e. from sidechain Monte Carlo sampling ${ }^{4}$ ) all of the relevant slow 
degrees of freedom. Second, there must be relatively few of these degrees of freedom so that deliberate sampling of them is tractable. In this particular binding site, crystallographic evidence suggests that the only sidechain reorientation on ligand binding is that of Val111 ${ }^{19}$, thus it is straightforward to apply this umbrella sampling approach. In general, however, umbrella sampling may prove impractical. But the confine-and-release approach itself (Figure 1) only requires a method of computing the confine and release free energies; this need not be done with umbrella sampling.

The confine-and-release cycle used here, involving confining and releasing the protein to compute true binding free energies, can easily be extended to a variety of other applications. In the example above, [virtual] confinement is due to kinetic trapping. But deliberate confinement by external restraints may also be useful. This could help, for example, for proteins that undergo relatively large conformational changes on ligand binding, such flap closure in HIV protease. Without this confinement, the protein could begin to deform back to its apo structure as the ligand is alchemically removed, leading to sampling problems. These sampling problems can be severe: At some alchemical intermediate states, both metastable states could be relevant, and the protein would need to sample both several times during the simulation. In HIV protease, for example, these conformational changes may take place on the microsecond to millisecond timescale and are difficult to sample even with long molecular dynamics trajectories ${ }^{18}$. Thus, this confinement approach can also potentially aid convergence at intermediate alchemical states.

We conclude that computing binding free energies requires more than just computing the binding free energy of the ligand to a particular conformational state of the protein; it also requires a calculation of the free energy associated with confining the protein to that particular conformational state with and without the ligand present. These confinement free energies can be substantial, even for the relatively rigid binding site considered here. Elsewhere, we have noted that similar problems can arise when sampling ligand orientations ${ }^{14}$. Unless free energy calculations include sufficient sampling to adequately include these conformational changes at all stages of the transformation, computed "binding free energies" are not true binding free energies. In short, a dependence of free energy estimates on initial protein or ligand structure can indicate that simulations are not adequately sampling the relevant regions of configuration space. The confine-and-release framework we introduce here can be used to design approaches that isolate and solve these sampling problems in a systematic and controlled manner for free energy calculations.

The importance of conformational change in binding free energies has ramifications that extend beyond just alchemical free energy calculations. Virtual screening methods that rely on docking and scoring to a single structure need to reconsider the assumption that binding free energies can be estimated given an appropriate bound structure. Free energy costs associated with trapping the protein to the holo structure, or to any structure chosen, may be significant, and probably need to be correctly accounted for to accurately predict binding free energies. This problem cannot be avoided simply by comparing relative binding free energies of different ligands, either. In this binding site, for example, it is known that some ligands bind without reorientation of the Val111 sidechain, while others require the reorientation seen here in the case of $p$-xylene ${ }^{19}$. This means that free energy costs required to bind different ligands can be substantially different - up to several $\mathrm{kcal} / \mathrm{mol}$, based on the data presented here. Thus, when estimating relative binding free energies using the same protein structure, errors will be different for different ligands rather than canceling out.

In summary, the confine-and-release framework presented here provides a rigorous way to correct for inadequate or restricted computational sampling of protein degrees of freedom in ligand binding free energy calculations. This approach can give binding free energies that are 
independent of the starting protein structure (i.e. apo or holo) and therefore yield true binding free energies for the given the force field. Here we have demonstrated this approach using an umbrella sampling technique for computing the confine-and-release free energies; sampling requirements will probably limit this particular technique to accounting for inadequate sampling of a limited number of degrees of freedom. But the framework is more general.

\section{Supplementary Material}

Refer to Web version on PubMed Central for supplementary material.

\section{Acknowledgment}

We thank Benoit Roux (University of Chicago) for a critical reading of the manuscript. JDC was supported in part by HHMI and IBM predoctoral fellowships. DLM and KAD acknowledge NIH grant GM63592, Anteon Corporation grant USAF-5408-04-SC-0008, and a UCSF Sandler Award.

\section{References}

1. Jorgensen WL. The Many Roles of Computation in Drug Discovery. Science 2004;303:1813-1818. [PubMed: 15031495]

2. McGovern SL, Shoichet BK. Information Decay in Molecular Docking Screens Against Holo, Apo, and Modeled Conformations of Enzymes. J. Med. Chem 2003;46:2895-2907. [PubMed: 12825931]

3. Huang N, Kalyanaraman C, Irwin JJ, Jacobson MP. Molecular Mechanics Methods for Predicting Protein-Ligand Binding. J. Chem. Inf. Model 2006;46:243-253. [PubMed: 16426060]

4. Meiler J, Baker D. ROSETTALIGAND: Protein-Small Molecule Docking with Full Side-Chain Flexibility. Prot.: Struct., Funct., Bioinf 2006;66:538-548.

5. Wang J, Deng Y, Roux B. Absolute Binding Free Energy Calculations Using Molecular Dynamics Simulations with Restraining Potentials. Biophys. J 2006;91:2798-2814. [PubMed: 16844742]

6. Shirts, MR. Ph.D. Dissertation. Stanford University; 2004 [accessed Feb. 1, 2007]. Calculating Precise and Accurate Free Energies in Biomolecular Simulations. ProQuest location: http://wwwlib.umi.com/dissertations/fullcit/3153076"

7. Fujitani H, Tanida Y, Ito M, Jayachandran G, Snow CD, Shirts MR, Sorin EJ, Pande VS. Direct Calculation of the Binding Free Energies of FKBP Ligands. J. Chem. Phys 2005;123:084108. [PubMed: 16164283]

8. Deng Y, Roux B. Calculation of Standard Binding Free Energies: Aromatic Molecules in the T4 Lysozyme L99A Mutant. J. Chem. Theor. and Comput 2006;2:1255-1273.

9. van den Bosch M, Swart M, Snijderst JG, Berendsen HJC, Mark AE, Oostenbrink C, van Gunsteren WF, Canters GW. Calculation of the Redox Potential of the Protein Azurin and Some Mutants. Chem. Bio. Chem 2005;6:738-746.

10. Zhou Y, Oostenbrink C, Jongejan A, Hagen WR, de Leeuw SW, Jongejan JA. Computational Study of Ground-State Chiral Induction in Small Peptides: Comparison of the Relative Stability of Selected Amino Acid Dimers and Oligomers in Homochiral and Heterochiral Combinations. J. Comp. Chem 2006;27:857-867. [PubMed: 16541426]

11. Leitgeb M, Schroder C, Boresch S. Alchemical Free Energy Calculations and Multiple Conformational Substates. J. Chem. Phys 2005;122:084109.

12. Gilson MK, Given JA, Bush GL, McCammon JA. The Statistical-Thermodynamic Basis for Computation of Binding Affinities: A Critical Review. Biophys. J 1997;72:1047-1069. [PubMed: 9138555]

13. Boresch S, Tettinger F, Leitgeb M, Karplus M. Absolute Binding Free Energies: A Quantitative Approach for Their Calculation. J. Phys. Chem. B 2003;107:9535-9551.

14. Mobley DL, Chodera JD, Dill KA. On the Use of Orientational Restraints and Symmetry Number Corrections in Alchemical Free Energy Calculations. J. Chem. Phys 2006;125:084902. [PubMed: 16965052] 
15. Straatsma TP, McCammon JA. Treatment of Rotational Isomers in Free Energy Evaluations. Analysis of the Evaluation of Free Energy Differences by Molecular Dynamiccs Simulations of Systems with Rotational Isomeric States. J. Chem. Phys 1989;90:3300-3304.

16. Essmann U, Perera L, Berkowitz M, Darden T, Lee H, Pedersen Lee GA. Smooth Particle Mesh Ewald Method. J. Chem. Phys 1995;103:8577-8593.

17. Kumar SJ, Rosenberg M, Bouzida D, Swendsen RH, Kollman PA. The Weighted Histogram Analaysis Method for Free-Energy Calculations on Biomolecules. I. The Method. J. Comp. Chem 1992;13:1011-1021.

18. Hornak V, Okur A, Rizzo RC, Simmerling C. HIV-1 Protease Flaps Spontaneously Open and Reclose in Molecular Dynamics Simulations. Proc. Nat. Acad. Sci. USA 2006;103:915-920. [PubMed: 16418268]

19. Morton A, Matthews BW. Specificity of Ligand Binding in a Buried Nonpolar Cavity of T4 Lysozyme: Linkage of Dynamics and Structural Plasticity. Biochemistry 1995;34:8576-8588. [PubMed: 7612599] 

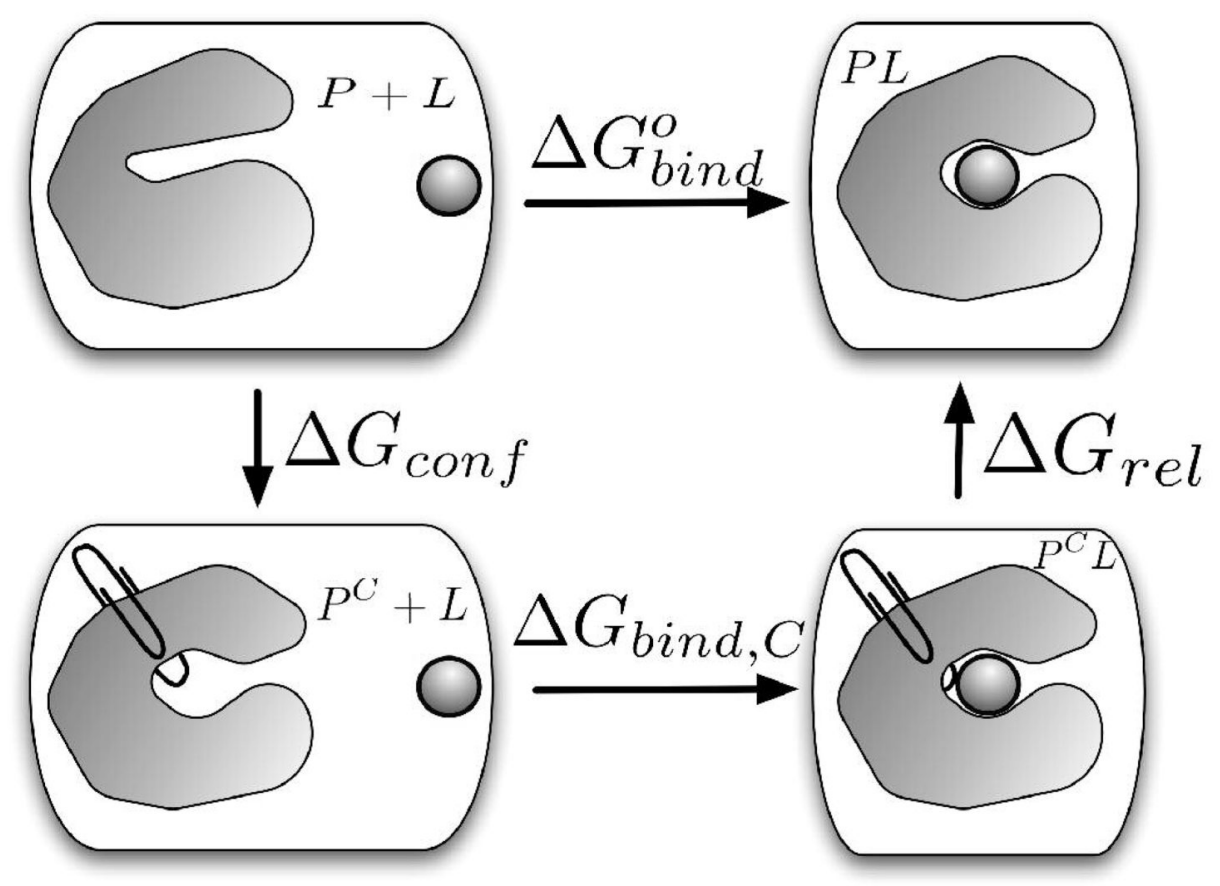

Figure 1.

Thermodynamic cycle for the confine-and-release framework. The quantity we want to calculate is $\Delta G_{\text {bind }}^{o}$ (top), the free energy difference for the process $P+L \rightarrow P L$. Kinetic trapping (virtual confinement) or deliberate confinement can keep conformational changes from being sampled (shown graphically by a paperclip). When this happens, computed free energies are actually confined binding free energies, $\Delta G_{\text {bind, } C}^{o}$ (bottom arrow). To relate these to true binding free energies, it is necessary to compute the free energy of confining the protein in the absence of the ligand (left arrow), and releasing the protein in the presence of the ligand (right arrow). 


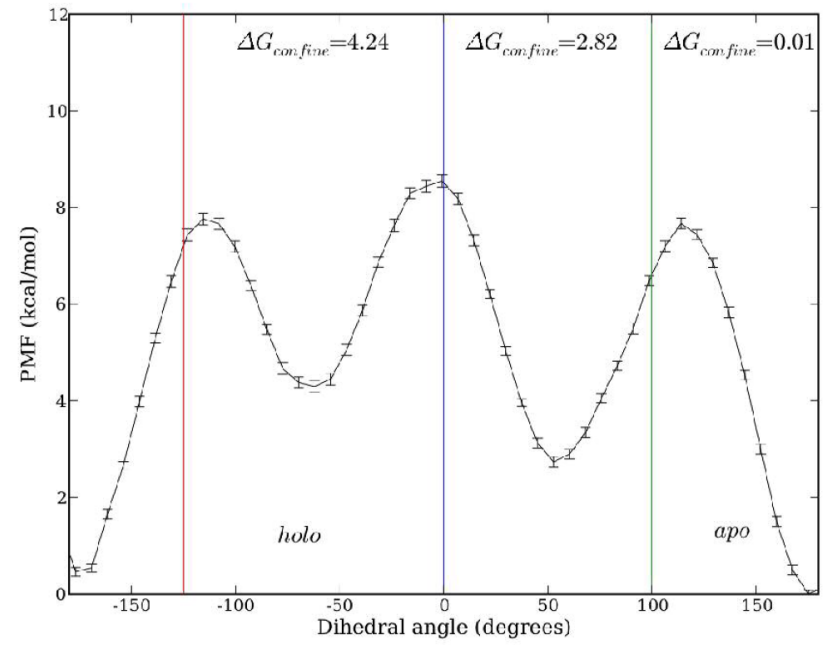

(a)

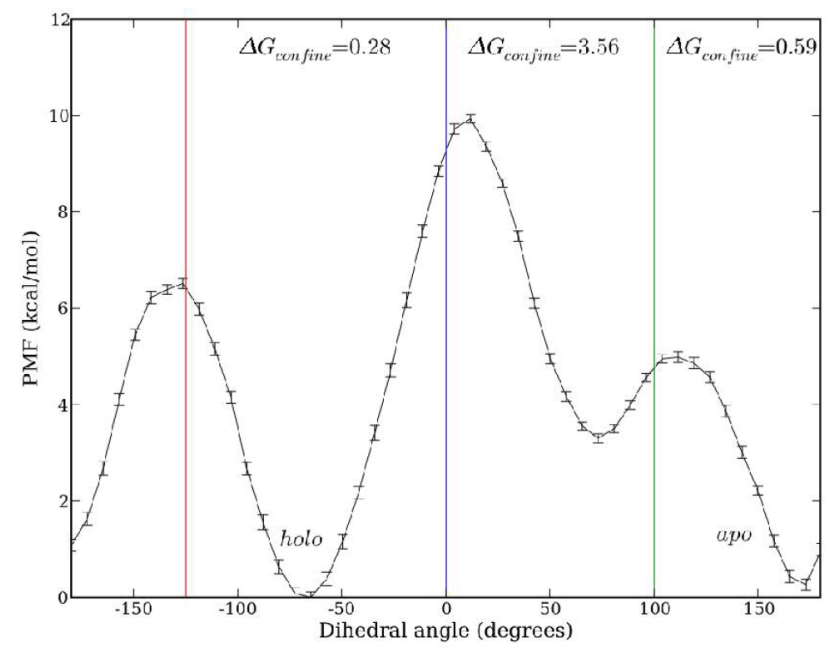

(b)

Figure 2.

Potential of mean force for rotating the valine 111 sidechain, with (b) and without (a) the ligand. Above each of the three regions is shown the free energy of confining Val111 to that metastable state. The apo metastable state corresponds to the first region on the left and the far right region (since the dihedral angle is periodic). Error bars represent statistical uncertainties corresponding to one standard deviation. Uncertainties for confinement to each well are given in the text. 